\title{
ON THE EXTREMALS OF THE PÓLYA-SZEGÖ INEQUALITY
}

\author{
ALMUT BURCHARD AND ADELE FERONE
}

\begin{abstract}
The distance of an extremal of the Pólya-Szegö inequality from a translate of its symmetric decreasing rearrangement is controlled by the measure of the set of critical points.
\end{abstract}

\section{INTRODUCTION}

Let $u$ be a nonnegative function on $\mathbb{R}^{n}$ that vanishes at infinity. Many geometric inequalities relate $u$ with its symmetric decreasing rearrangement, $u^{\star}$. The Pólya-Szegö inequality states that

$$
\left\|\nabla u^{\star}\right\|_{p} \leq\|\nabla u\|_{p}
$$

for every $1 \leq p \leq \infty$ such that the distributional gradient $|\nabla u|$ lies in $L^{p}$; in particular, $\left|\nabla u^{\star}\right|$ again lies in $L^{p}[15]$. For $p=1$, this reduces to the isoperimetric inequality, and for $p=\infty$, it follows from the fact that symmetric decreasing rearrangement improves the modulus of continuity.

Inequality (1.1) has been extended in various directions. It holds for general convex Dirichlet-type functionals [5, 16], on the larger space of functions that are locally of bounded variation [8], and with other symmetrizations in place of the symmetric decreasing rearrangement $[2,13$, $6,4]$. The functionals that satisfy general Pólya-Szegö inequalities have been fully characterized; they are known to include all rearrangementinvariant norms [7, Theorem 1.2].

In this paper, we study functions that produce equality in (1.1) for some $p$ with $1<p<\infty$. Such a function will be called an extremal of the inequality.

Extremals of (1.1) were first analyzed by Brothers and Ziemer in 1988 [5]. Clearly, every translate of a symmetric decreasing function is an extremal. In the converse direction, the level sets of extremals must be balls, but they need not be concentric. For example, a function whose graph consists of a small cone stacked on the frustrum of a large cone is an extremal, regardless of the precise position of the smaller cone on the plateau. Brothers and Ziemer discovered that a similar phenomenon can occur even for functions without plateaus. Under the 
assumption that the distribution function of $u$ is absolutely continuous, they proved that the only extremals are translates of $u^{\star}$. Otherwise, there exist extremals that are equimeasurable to, but not translates of $u^{\star}$.

The condition that the distribution function be absolutely continuous is equivalent to requiring that the set of non-trivial critical points of $u^{\star}$ has measure zero. What can be said about extremals where this set has positive measure? In 2006, Cianchi and Fusco proved that every extremal of (1.1) whose support has finite measure satisfies

$$
\left\|u-u^{\star} \circ \tau\right\|_{1} \leq L_{n}\|\nabla u\|_{p} \cdot \lambda_{n}(\operatorname{supp} u)^{\frac{1}{p^{\prime}}+\frac{2 n-1}{2 n^{2}}} \lambda_{n}(C)^{\frac{1}{2 n^{2}}}
$$

for a suitable translation $\tau\left[9\right.$, Theorem 1.1]. Here, $L_{n}$ is a constant that depends on the dimension, $\lambda_{n}$ is the $n$-dimensional Lebesgue measure, $p^{\prime}=p /(p-1)$ is the Hölder dual exponent of $p$, and $C$ is the set of critical points defined in Theorem 1 below. Our goal is to simplify the analysis and construct explicit bounds for extremals whose support need not have finite measure.

The results of Brothers-Ziemer and Cianchi-Fusco apply to certain convex Dirichlet-type functionals that will be described below, and to the more general functionals treated in [7], which need not be in integral form. They remain valid - after adjusting the constants for the convex rearrangement, which replaces the level sets of $u$ by suitably scaled copies of a centrally symmetric convex body $B \subset \mathbb{R}^{n}$. For the sake of simplicity, we focus on the classical case of the $L^{p}$-norm of the gradient with $1<p<\infty$, leaving the discussion of more general functionals for the last section of the paper.

Theorem 1. Let $u$ be a nonnegative function on $\mathbb{R}^{n}$ that vanishes at infinity and whose distributional gradient lies in $L^{p}$ for some $1<p<$ $\infty$, and let $u^{\star}$ be its symmetric decreasing rearrangement. If

$$
\|\nabla u\|_{p}=\left\|\nabla u^{\star}\right\|_{p}
$$

then there exists a translation $\tau$ such that

$$
\left\|u-u^{\star} \circ \tau\right\|_{q} \leq K_{n}^{1 / q}\|u\|_{q}^{1 / n^{\prime}}\left\|u \mathcal{X}_{C}\right\|_{q}^{1 / n}
$$

for every $q \geq 1$ with $u \in L^{q}$. Here, $K_{n}=2 \omega_{n-1} / \omega_{n}$, and

$$
C=\left\{x \in \mathbb{R}^{n}: 0<u(x)<\operatorname{ess} \sup u \text { and }|\nabla u(x)|=0\right\} .
$$

The translation $\tau$ is chosen to align the graphs of $u^{\star} \circ \tau$ and $u$ at the top. The value of the constant is given by $K_{n}=\left(\int_{0}^{\pi / 2} \cos ^{n} \theta d \theta\right)^{-1} \sim$ $(2 n / \pi)^{1 / 2}$. The set $C$ consists of the non-trivial critical points of $u$, except for the possible plateau at height ess $\sup u$. For the conclusion of the theorem, we can equivalently replace the function $u$ by $u^{\star}$ on the 
right hand side of (1.3) and in the definition of $C$. Indeed, if $u \in W_{l o c}^{1,1}$ and $S$ is the set where the singular part of the distribution function is concentrated, then $C \supset u^{-1}(S)$ in general, and equality holds if $u$ is an extremal.

If $C$ has finite measure, there is a simpler estimate in terms of its volume radius.

Theorem 2. Under the assumptions of Theorem 1, if $\lambda_{n}(C)<\infty$ then there exists a translation $\tau$ such that

$$
\left\|u-u^{\star} \circ \tau\right\|_{p} \leq\|\nabla u\|_{p} \cdot\left(\frac{\lambda_{n}(C)}{\omega_{n}}\right)^{\frac{1}{n}} .
$$

For $1<p<n$, a natural choice for $q$ in Theorem 1 is the Sobolev exponent $p^{*}=n p /(n-p)$, for which the right hand side of (1.3) is bounded by the Sobolev inequality. Interpolating with (1.4) yields $L^{q}$ bounds for $p<q<p^{*}$, provided that $C$ has finite measure. For $p>n$, there is a corresponding bound in $L^{\infty}$.

Theorem 3. Under the assumptions of Theorem 1, if $p>n$ and $\lambda_{n}(C)<\infty$ then there exists a translation $\tau$ such that

$$
\left\|u-u^{\star} \circ \tau\right\|_{\infty} \leq M_{n, p}\|\nabla u\|_{p} \cdot\left(\frac{\lambda_{n}(C)}{\omega_{n}}\right)^{\frac{1}{n}-\frac{1}{p}}
$$

where $M_{n, p}$ is the Morrey constant.

We briefly describe the relation with the literature. Theorem 1 contains the result of Brothers and Ziemer, because the right hand side of (1.3) vanishes when the distribution function of $u$ is absolutely continuous. Similarly, Theorem 2 contains the bound of Cianchi and Fusco. To see this, apply Hölder's inequality on the left hand side of (1.4) and use that $C \subset \operatorname{supp} u$ on the right to obtain (1.2) with $L_{n}=2^{1 / p^{\prime}} \omega_{n}^{-1 / n}$. We will show below that the proof of Theorem 1 also implies (1.2). However, Theorems 2 and 3 do not seem to follow directly from Theorem 1.

Acknowledgments. Research for this paper was supported in part by an NSERC Discovery Grant and a GNAMPA Project.

\section{Outline OF The PRoOF}

Brothers and Ziemer characterized extremals as follows. If $u$ satisfies the assumptions of Theorem 1, then its level sets are balls,

$$
\{u>t\}=\xi_{t}+\left\{u^{\star}>t\right\}
$$


(up to sets of Lebesgue measure zero). Furthermore, the gradient is equidistributed on level sets,

$$
|\nabla u(x)|_{\lrcorner \partial\{u>t\}}=\left|\nabla u^{\star}\right|_{\lrcorner \partial\left\{u^{\star}>t\right\}}
$$

for $\mathcal{H}^{n-1}$-almost every $x \in \partial\{u>t\}$ and almost every $t \in(0$, ess $\sup u)$. This equidistribution property is a consequence of the strict convexity of the function $t \rightarrow t^{p}$. All later work on the problem relies on this characterization.

A more delicate issue is to prove that the level sets are concentric balls if the distribution function of $u$ is absolutely continuous. Brothers and Ziemer, starting from (2.1), express $u^{\star}$ in terms of $u$ as $u^{\star}=u \circ T$ and study the regularity of the transformation $T$ under the assumption of the continuity of the distribution function. The crucial point is the evaluation of $\nabla u^{\star}$, which requires a non-standard chain rule because $u$ is just a Sobolev function. The absolute continuity of the distribution function is needed to deduce that $T$ is a translation of the identity map from (2.2) and the fact that $\nabla u^{\star}(x)=(D T(x))^{t} \nabla u(T(x))$.

In the last ten years, several new proofs of these results have appeared. In [11], the authors reverse the approach of Brothers and Ziemer and express $u$ in terms of $u^{\star}$ as $u(x)=u^{\star}(T(x))$. That leads to an easier case of the chain rule, because $u^{\star}$ is essentially a function of a single variable. Finally, the conclusion is obtained by a gradient-flow argument. Since this last part of the proof relies on the uniform convexity and smoothness properties of the Euclidean norm, the authors later developed yet another geometric argument to treat rearrangements with respect to arbitrary norms in $\mathbb{R}^{n}[12]$. Their method was subsequently used by Cianchi and Fusco in [9].

The argument in [12] proceeds as follows. Let $\xi_{t}$ denote the center of the ball $\{u>t\}$, and let $R(x)$ be the function that assigns to each point $x \in \mathbb{R}^{n}$ the radius of the ball $\{u>u(x)\}$. The key observation is that for all $s, t \in(0, \operatorname{ess} \sup u)$ there exists a pair of points $x \in \partial\{u>s\}$ and $y \in \partial\{u>t\}$ such that

$$
\left|\xi_{s}-\xi_{t}\right|=|R(x)-R(y)|-|x-y|,
$$

see Fig. 1. If the distribution function of $u$ is absolutely continuous, then $R$ is Lipschitz continuous and $|\nabla R| \equiv 1$. It follows that $\xi_{t}$ is constant, proving that $u$ is a translate of $u^{\star}$.

If the distribution function of $u$ is not absolutely continuous, then $R$ is of bounded variation. In [9], the distribution function of $u$ is approximated by an absolutely continuous function. Instead, we approximate $u$ by functions whose distribution functions have jumps, but no singular continuous part. In Section 4, we analyze the variation of $R$ for 


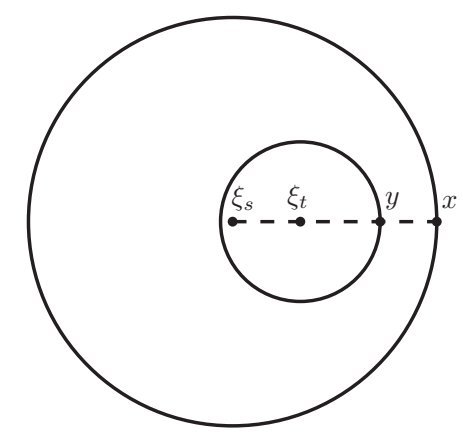

FiguRE 1. Two circles ordered by inclusion. The difference between the radii can be expressed as the sum of their distance $|x-y|$ and the distance of the centers $\left|\xi_{s}-\xi_{t}\right|$, see Eq. (2.3).

such functions and derive the bound

$$
\left|\xi_{s}-\xi_{t}\right| \leq\left(\frac{1}{\omega_{n}} \mu^{s}((s, t])\right)^{\frac{1}{n}}
$$

where $\mu^{s}$ is the singular part of the measure associated with the distribution function of $u$. It follows that the total variation of $\xi$ is bounded by the volume radius of $C$,

$$
\|D \xi\| \leq\left(\frac{\lambda_{n}(C)}{\omega_{n}}\right)^{\frac{1}{n}} .
$$

In Section 5, we show that this implies the main results. The final Section 6 is dedicated to convex Dirichlet functionals.

Before turning to the technical part, observe that the characterization of extremals given by Brothers and Ziemer does not depend on the value of $p$. If $u$ is an extremal for some $1<p<\infty$, then (2.1)(2.2) imply by the coarea formula that $u$ produces equality in (1.1) for every $1 \leq p \leq \infty$. In fact, much more is true. According to [7, Theorem 1.7], there is a wide class of functionals satisfying a suitable strict monotonicity condition that have the same family of extremals, which all satisfy the conclusions of Theorem 1-3 (as well as those of Corollaries 6.1-6.2 with $V=\emptyset$ ).

\section{Notation AND PRELIMINARY RESUlts}

We work on $\mathbb{R}^{n}$, equipped with the standard Euclidean norm $|\cdot|$ and Lebesgue measure $\lambda_{n}$. Let $u$ be a nonnegative measurable function on $\mathbb{R}^{n}$. We always assume that $u$ vanishes at infinity, in the sense that 
its level sets $\left\{x \in \mathbb{R}^{n}: u(x)>t\right\}$ have finite measure for all $t>0$. Its distribution function is given by

$$
F(t)=\lambda_{n}\left(\left\{x \in \mathbb{R}^{n}:|u(x)|>t\right\}\right), \quad t>0 .
$$

The symmetric decreasing rearrangement of $u$ is defined by

$$
u^{\star}(x)=\sup \left\{t>0: F(t)>\omega_{n}|x|^{n}\right\} \quad x \in \mathbb{R}^{n} ;
$$

it is the unique radially decreasing function that is equimeasurable to $u$ and lower semicontinuous.

The following construction removes a collection of horizontal slices from the graphs of $u$ and $u^{\star}$ (see Fig. 2). Given a finite or countable union of intervals $I \subset \mathbb{R}_{+}$, set

$$
f(t)=\lambda_{1}([0, t] \backslash I) .
$$

Then $f \circ u$ vanishes at infinity, and $(f \circ u)^{\star}=f \circ u^{\star}$. If $u \in W_{\mathrm{loc}}^{1,1}$, then $f \circ u \in W_{\text {loc }}^{1,1}$, and

$$
\nabla(f \circ u)(x)=\mathcal{X}_{\{u(x) \notin I\}} \nabla u(x)
$$

almost everywhere on $\mathbb{R}^{n}$ (see [14, Corollary 6.18]).
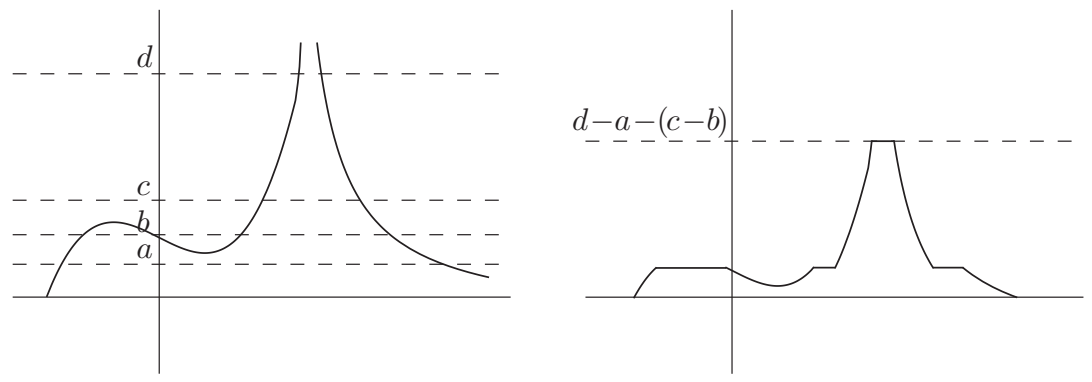

FIGURE 2. Removing a horizontal slice from the graph of $u$, see Eq. (3.2). The right panel shows $f \circ u$ with $I=[0, a) \cup$ $(b, c) \cup(d, \infty)$.

Many useful quantities can be expressed in terms of distribution functions. For any absolutely continuous function $\Psi$ on $\mathbb{R}_{+}$with $\Psi(0)=$ 0 , there is the layer-cake representation

$$
\int \Psi(u) d x=\int_{0}^{\infty} F(t) \Psi^{\prime}(t) d t .
$$

We note for later use that

$$
\|u-v\|_{1}=\int_{0}^{\infty} \lambda_{n}(\{u>t\} \triangle\{v>t\}) d t
$$


for any pair of nonnegative integrable functions (here $\triangle$ stands for the symmetric difference of sets). The following two lemmas provide similar formulas for other convex functions of $|u-v|$.

Lemma 3.1. Let $\Psi$ be a convex function on $\mathbb{R}_{+}$with $\Psi(0)=\Psi^{\prime}(0)=0$, let $\nu$ be the measure that represents its second distributional derivative, and let $u, v$ be nonnegative measurable functions. Then

$$
\int \Psi(|u-v|) d x=\int_{0}^{\infty} \int_{0}^{t}\left[\lambda_{n}(\{u>t\} \backslash\{v>t-s\})\right.
$$

$$
\left.+\lambda_{n}(\{v>t\} \backslash\{u>t-s\})\right] d \nu(s) d t .
$$

Proof. We use that

$$
\Psi(b-a)=\int_{a}^{b} \int_{0}^{t-a} d \nu(s) d t=\left(\nu \times \lambda_{1}\right)(\{(s, t): a+s<t<b\})
$$

for $b>a>0$, and split the integral according to the sign of $u-v$,

$$
\int \Psi(|u-v|) d x=\int_{\{v>u\}} \Psi(v-u) d x+\int_{\{u>v\}} \Psi(u-v) d x .
$$

For the first integral on the right, Fubini's theorem gives

$$
\begin{aligned}
\int_{\{v>u\}} \Psi(v-u) d x & =\left(\nu \times \lambda_{1} \times \lambda_{n}\right)(\{(s, t, x): u(x)+s<t<v(x)\}) \\
& =\int_{0}^{\infty} \int_{0}^{t} \lambda_{n}(\{v>t\} \backslash\{u>t-s\}) d \nu(s) d t .
\end{aligned}
$$

Treating the second integral in the same way, we arrive at the claimed identity.

Lemma 3.2. Let $\Psi$ be a convex function on $\mathbb{R}_{+}$with $\Psi(0)=0$, and let $u, v$ be nonnegative measurable functions. Then

$$
\int \Psi(|u-v|) d x \leq \int_{0}^{\infty} \lambda_{n}(\{u>t\} \triangle\{v>t\}) \Psi^{\prime}(t) d t .
$$

Proof. Since the claim holds for linear functions $\Psi$ by (3.3), we may assume, by replacing $\Psi(t)$ with $\Psi(t)-t \Psi^{\prime}\left(0_{+}\right)$that $\Psi^{\prime}(0)=0$. Then we can apply Lemma 3.1. The right hand side of (3.4) increases if we set $s=0$ in the integrand. We then evaluate the inner integral, using that $\nu(0, t)=\Psi^{\prime}(t)$ for almost every $t$.

Since the distribution function of $u$ is monotonically decreasing, it defines a Borel measure on $\mathbb{R}_{+}$by

$$
\mu((a, b])=F(a)-F(b)=\lambda_{n}\left(u^{-1}(a, b]\right) .
$$


If $u$ is essentially bounded, we restrict this measure to the interval $(0, \operatorname{ess} \sup u)$, neglecting plateaus at $t=0$ and $t=\operatorname{ess} \sup u$. Consider the Lebesgue-Radon-Nikodym decomposition $\mu=\mu^{a c}+\mu^{s}$, where $\mu^{a c} \ll \lambda_{1}$ and $\mu^{s} \perp \lambda_{1}$. This gives rise to a decomposition of the distribution function $F=F^{a c}+F^{s}$, where $F^{s}(t)=\mu^{s}(t$, ess sup $u)$ and $F^{a c}$ is absolutely continuous. By the Fundamental Theorem of Calculus, the density of $\mu^{a c}$ is given by the classical derivative

$$
-F^{\prime}(t)=\frac{\operatorname{Per}\left(\left\{u^{\star}>t\right\}\right)}{\left|\nabla u^{\star}\right|_{\rfloor \partial\left\{u^{\star}>t\right\}}},
$$

and the derivative of $F^{s}$ vanishes almost everywhere. The singular part of the measure is given by

$$
\mu^{s}((a, b])=F^{s}(a)-F^{s}(b)=\lambda_{n}\left(\left\{x \in\left(u^{\star}\right)^{-1}((a, b]): \nabla u^{\star}(x)=0\right\}\right),
$$

it is supported on the set of singular values

$$
S=\{t \in(0, \operatorname{ess} \sup u): F \text { is not differentiable at } t\} .
$$

Since $u^{\star}$ is a monotone function of the single radial variable, the set $S$ has measure zero. Clearly $\mu$ is absolutely continuous if and only if $\left(u^{\star}\right)^{-1}(S)$ has measure zero. Since the gradient of $u$ vanishes almost everywhere on $u^{-1}(S)\left[14\right.$, Theorem 6.19], its follows that $C \supset u^{-1}(S)$ up to a set of measure zero, and

$$
\mu^{s}((a, b]) \leq \lambda_{n}\left(\left\{x \in u^{-1}((a, b]): \nabla u(x)=0\right\}\right) .
$$

Note that strict inequality can occur: when equality holds in (3.7) $u$ is said to be coarea regular [1]. The next result shows that every extremal is coarea regular (see also $[6,8]$ ), and allows us to interpret $F^{s}(t)$ as the distribution function of the restriction of $u$ to $C$.

Lemma 3.3. Under the assumptions of Theorem 1, $C=u^{-1}(S)$ up to a set of measure zero. Furthermore,

$$
F^{s}(t)=\lambda_{n}(\{x \in C: u(x)>t\}) .
$$

Proof. Since $C \supset u^{-1}(S)$, we have only to prove the reverse inclusion. The coarea formula and the characterization of extremals in (2.1) and (2.2) show that

$$
\begin{aligned}
\lambda_{n}(\{x \notin C: u(x)>t\}) & =\int_{t}^{\infty} \int_{\partial\{u>t\}}|\nabla u|^{-1} d \mathcal{H}^{n-1} d t \\
& =\int_{t}^{\infty}\left|\nabla u^{\star}\right|^{-1} \operatorname{Per}\left(\left\{u^{\star}>t\right\}\right) d t \\
& =\lambda_{n}\left(\left\{x: \nabla u^{\star}(x) \neq 0, u^{\star}(x)>t\right\}\right) .
\end{aligned}
$$


Since $u$ and $u^{\star}$ are equimeasurable, it follows that

$$
\begin{aligned}
\lambda_{n}(\{x \in C: u(x)>t\}) & =\lambda_{n}\left(\left\{x: \nabla u^{\star}(x)=0, u^{\star}(x)>t\right\}\right) \\
& =F^{s}(t) .
\end{aligned}
$$

In general, $\mu^{s}$ can be further decomposed into a sum of (at most) countably many point masses that correspond to plateaus of $u$, and a singular continuous component. However, one can always approximate $u$ by functions whose distribution function has no singular continuous part.

Lemma 3.4. (Approximation) Let $u \in W_{\mathrm{loc}}^{1,1}$ be a nonnegative function that vanishes at infinity. There exists an increasing sequence of functions $u_{m} \in W_{\text {loc }}^{1,1}$ and a decreasing sequence of sets $C_{m} \subset \mathbb{R}^{n}$ with

$$
\lim u_{m}=u, \quad \bigcap C_{m}=u^{-1}(S),
$$

such that $u_{m}$ is bounded and supported on a set of finite measure, each level set $\left\{u_{m}>t\right\}$ is also a level set of $u$, the distribution function of $u_{m}$ has no singular continuous part, and

$$
\nabla u_{m}=\mathcal{X}_{C_{m}} \nabla u, \quad(m \geq 1) .
$$

Proof. Since $\mu^{s}$ is a regular Borel measure, there exists a decreasing sequence of open sets $S_{m}$ containing $S$ such that

$$
\lim \mu\left(S_{m} \cap(t, \operatorname{ess} \sup u)\right)=\mu^{s}(t, \operatorname{ess} \sup u)
$$

for all $t>0$. If $u$ is unbounded or the support of $u$ does not have finite measure, we ask that $S_{m} \supset[0,1 / m) \cup(m, \infty)$. Set $f_{m}(t)=$ $\lambda_{1}\left([0, t] \backslash S_{m}\right)$, let $u_{m}=f_{m} \circ u$, and let $C_{m}=u^{-1}\left(S_{m}\right)$. Then $u_{m}$ is bounded and supported on a set of finite measure. Moreover, since $S_{m}$ is open, it is the union of (at most) countably many disjoint intervals. Therefore

$$
\nabla u_{m}(x)=\mathcal{X}_{u(x) \notin S_{m}} \nabla u(x)
$$

for almost every $x$. By construction, $u_{m}$ increases monotonically to $u$, and $\left|\nabla u_{m}\right|$ increases to $|\nabla u|$. For each connected component $(a, b)$ of $S_{m}$, the distribution function of $u_{m}$ has a jump of size $F(a)-F(b)$, corresponding to a plateau of $u_{m}^{\star}$. Since $S_{m} \supset S$, the distribution function of $u_{m}$ has no singular continuous component.

We will also consider functions on $\mathbb{R}^{n}$ that do not lie in $W_{\text {loc }}^{1,1}$ but in the larger space $B V_{\text {loc }}$. A function $u$ is locally of bounded variation, if its distributional derivative is represented by a vector-valued Radon measure, $[D u]$. We denote by $|D u|$ the corresponding variation measure, and by $\|D u\|=|D u|\left(\mathbb{R}^{n}\right)$ its total variation. The variation measure has Lebesgue-Radon-Nikodym representation $[D u]=$ 
$\left[D^{a c} u\right]+\left[D^{s} u\right]$, where the absolutely continuous component has density $\nabla u$, and $\left[D^{s} u\right]$ is supported on a set of Lebesgue measure zero. We will always use the precise representative of $u$ that agrees with its Lebesgue density limit at every point where it exists. For more information about $B V_{\text {loc }}$, we refer the reader to to $[10,3]$.

If $u$ is a nonnegative function in $B V_{\text {loc }}$ that vanishes at infinity, then $u^{\star} \in B V_{\text {loc }}$. Since $u^{\star}$ is a monotone function of the radius, its singular continuous component is supported on $\left(u^{\star}\right)^{-1}(\{t \in(0, \operatorname{ess} \sup u)$ : $\left.\left.F^{\prime}(t)=0\right\}\right)$.

\section{Properties of extremals}

Throughout this section, we assume that $u$ is an extremal for the Pólya-Szegő inequality (1.1). The goal is to prove the bounds on the variation of $\xi$ in (2.4) and (2.5).

Let $R$ be the function that assigns to each point $x \in \mathbb{R}^{n}$ the radius of the level set of $u$ at height $u(x)$,

$$
R(x)=\left(\frac{1}{\omega_{n}} F(u(x))\right)^{\frac{1}{n}} .
$$

Since $u^{\star}$ is a radial function, we can write $u=u^{\star} \circ T$, where $T(x)=$ $R(x) \cdot x /|x|$, as in $[11,12]$. The next two lemmas provide a bound on $|R(x)-R(y)|$.

Lemma 4.1. Under the assumptions of Theorem 1, if the support of $u$ has finite measure then $R$ is of bounded variation. The absolutely continuous part of its variation has density $\nabla R$, where

$$
|\nabla R|(x)= \begin{cases}1 & \text { if } u(x) \notin S \text { and } 0<u(x)<\operatorname{ess} \sup u \\ 0 & \text { otherwise }\end{cases}
$$

for almost every $x$.

Proof. The total variation of $R$, given by

$$
\|D R\|=\int_{0}^{\infty} \operatorname{Per}(\{R<t\}) d t
$$

is finite, because its value is bounded by the radius of the support of $u$ and its (sub-) level sets $\{R<t\}$ are smaller balls.

The distributional derivative of $R$ is represented by the vector-valued measure $[D R]$. For its absolutely continuous component, the chain rule yields on $u^{-1}((0, \operatorname{ess} \sup u) \backslash S)$

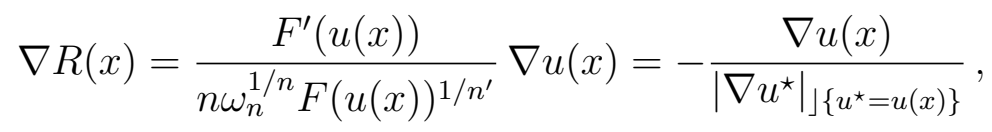


see [12, Eqs.(3.8)-(3.10)]. Here, $F^{\prime}(t)$ is the classical derivative of $F$, and we have used (3.6) in the second step. Since $u$ is an extremal, we see from (2.2) that the denominator agrees with $|\nabla u(x)|$, and therefore $|\nabla R|=1$ almost everywhere on $u^{-1}((0, \operatorname{ess} \sup u) \backslash S)$. Since $\lambda_{1}(S)=0$, the gradient vanishes almost everywhere on $u^{-1}(S)$.

Lemma 4.2. Under the assumptions of Theorem 1,

$$
|R(x)-R(y)| \leq|x-y|+\left(\frac { 1 } { \omega _ { n } } \left(\mu^{s}((u(x), u(y)])^{\frac{1}{n}}\right.\right.
$$

for almost every $x, y$ with $u(x)<u(y)$.

Proof. By Lemma 3.4, it suffices to consider functions $u$ whose support has finite measure and whose distribution function has no singular continuous component. Let

$$
r(\theta)=R(\theta x+(1-\theta) y), \quad 0 \leq \theta \leq 1
$$

be the restriction of $R$ to the line segment that joins $y$ with $x$. Since we choose for $R$ its precise representative in $B V_{\text {loc }}$, the restriction is of bounded variation and the chain rule holds for almost every choice of $x, y$ and almost every $\theta[3$, Theorem 3.107]. By Lemma 4.1, we have

$$
\left|r^{\prime}(\theta)\right|=|\langle\nabla R(\theta x+(1-\theta) y), x-y\rangle| \leq 1,
$$

and obtain for the absolutely continuous part

$$
r^{a c}(1)-r^{a c}(0) \leq|x-y| .
$$
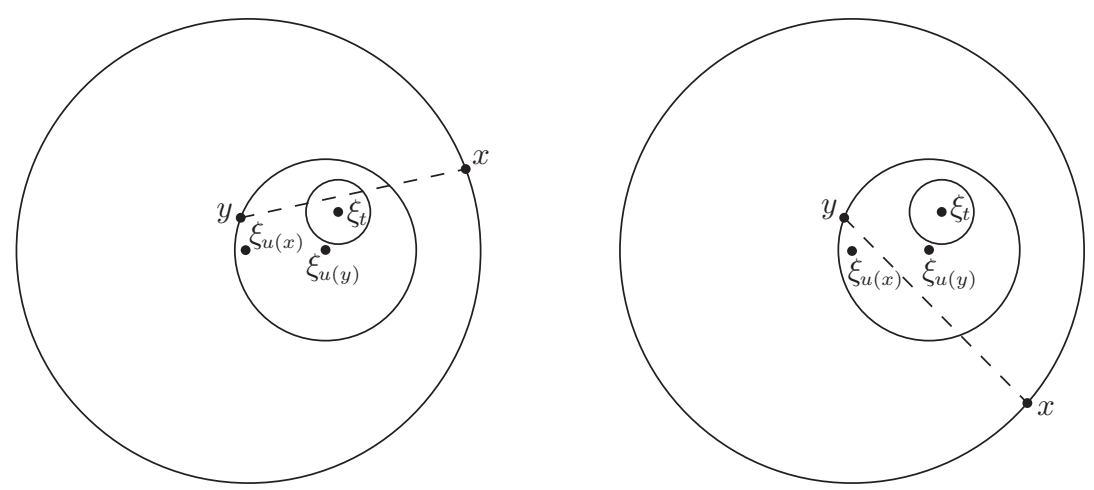

Figure 3 . The line segment from $y$ to $x$ crosses the boundary of a higher level set either twice (left) or never (right).

For the singular part, recall that $u(x)<u(y)$, and thus $R(x)>R(y)$. The line segment enters each level set $\{u>t\}$ with $t \in(u(x), u(y)$ ] exactly once; the boundary of a level set outside this range is crossed 
either twice, in opposite directions, or not at all, see Fig. 3. When the line segment enters $\{u>t\}$ for some $t \in S$, then $R$ experiences a positive jump of size $\omega_{n}^{-1 / n}\left(F\left(t_{-}\right)^{1 / n}-F(t)^{1 / n}\right)$; the jump is reversed upon exit. Since $S$ is countable, this yields

$$
\begin{aligned}
r^{s}(1)-r^{s}(0) & =\omega_{n}^{-\frac{1}{n}} \sum_{t \in S \cap(u(x), u(y)]}\left(F\left(t_{-}\right)^{\frac{1}{n}}-F(t)^{\frac{1}{n}}\right) \\
& =\omega_{n}^{-\frac{1}{n}} \sum_{t \in S \cap(u(x), u(y)]} \int_{F\left(t_{-}\right)}^{F(t)} \frac{1}{n} s^{\frac{1}{n}-1} d s \\
& \leq\left(\frac{1}{\omega_{n}} \sum_{t \in S \cap(u(x), u(y)]}\left(F\left(t_{-}\right)-F(t)\right)\right)^{\frac{1}{n}} .
\end{aligned}
$$

We have used that the intervals $\left(F\left(t_{-}\right), F(t)\right)$ are disjoint and that the function $s \mapsto s^{1-1 / n}$ is decreasing to move the domain of integration to the origin. By definition, the last sum equals $\mu^{s}((u(x), u(y)])$. The claim follows by adding the inequalities for $r^{a c}$ and $r^{s}$.

Proof of (2.4)-(2.5). Insert Lemma 4.2 into (2.3) to obtain the bound on $\left|\xi_{s}-\xi_{t}\right|$. The bound on the total variation follows by maximizing over $s, t$ and using Lemma 3.3.

We have used Lemmas 4.1 and 4.2 to show that the total variation of $\xi$ is bounded by $\left(\left\|D^{s} F\right\| / \omega_{n}\right)^{1 / n}$. The proof of Lemma 4.2 yields the somewhat stronger statement that

$$
\|D \xi\| \leq\left\|D^{s}\left(\frac{F}{\omega_{n}}\right)^{1 / n}\right\| .
$$

A similar computation as in Lemma 4.2 shows that the singular part of the variation of $R$ is bounded by the measure of the set of critical points,

$$
\left\|D^{s} R\right\| \leq \mu^{s}((0, \operatorname{ess} \sup u))+\inf _{t<\operatorname{ess} \sup u} F(t) .
$$

Here, the last term on the right represents the possible plateau at the top, which does not contribute to the variation of $\xi$.

\section{Proof of the MAIN Results}

Proof of Theorem 1. Since $u$ is an extremal, each level set $\{u>t\}$ is a ball centered at $\xi_{t}$. Let $\xi_{\infty}$ be the center of the ball

$$
\bigcap_{t \in(0, \operatorname{ess} \sup u)}\{u>t\}
$$


(which may consist of a single point), and consider the translation $\tau(x)=x-\xi_{\infty}$. By (2.3) and Lemma 4.2, the distance between the level sets $\{u>t\}$ and $\left\{u^{\star} \circ \tau>t\right\}$ is bounded by

$$
\left|\xi_{t}-\xi_{\infty}\right| \leq\left(\frac{F^{s}(t)}{\omega_{n}}\right)^{\frac{1}{n}}
$$

Since the symmetric difference between two balls of equal radius in $\mathbb{R}^{n}$ satisfies

$$
\lambda_{n}((\xi+B) \triangle(\eta+B)) \leq 2 \omega_{n-1}\left(\frac{\lambda_{n}(B)}{\omega_{n}}\right)^{\frac{1}{n^{\prime}}} \cdot|\xi-\eta|,
$$

it follows that

$$
\lambda_{n}\left(\{u>t\} \triangle\left\{u^{\star} \circ \tau>t\right\}\right) \leq K_{n} F(t)^{\frac{1}{n^{\prime}}} F^{s}(t)^{\frac{1}{n}},
$$

where $K_{n}=2 \omega_{n-1} / \omega_{n}$. From Lemma 3.2 with $\Psi(t)=t^{q}$, we deduce

$$
\begin{aligned}
\left\|u-u^{\star} \circ \tau\right\|_{q}^{q} & \leq K_{n} \int_{0}^{\infty} F(t)^{\frac{1}{n^{\prime}}} F^{s}(t)^{\frac{1}{n}} q t^{q-1} d t \\
& \leq K_{n}\left(\int_{0}^{\infty} F(t) q t^{q-1} d t\right)^{\frac{1}{n^{\prime}}}\left(\int_{0}^{\infty} F^{s}(t) q t^{q-1} d t\right)^{\frac{1}{n}} \\
& =K_{n}\|u\|_{q}^{q / n^{\prime}}\left\|u \mathcal{X}_{C}\right\|_{q}^{q / n} .
\end{aligned}
$$

We have applied Hölder's inequality with exponents $1 / n^{\prime}$ and $1 / n$, and used Lemma 3.3 to interpret $F^{s}$ as the distribution function of $u \mathcal{X}_{C}$.

The basic estimate in the first line of (5.2) can be used to derive other bounds on $u-u^{\star} \circ \tau$, for example

$$
\frac{\left\|u-u^{\star} \circ \tau\right\|_{q}^{q}}{\|u\|_{q}^{q}} \leq K_{n} \sup _{0<t<\operatorname{esssup} u}\left(\frac{\lambda_{n}(C \cap\{u>t\})}{\lambda_{n}(\{u>t\})}\right)^{\frac{1}{n}} .
$$

The ratio on the right hand side can be viewed as the density of $C$ in the level set $\{u>t\}$. It is always strictly less than one, because $C$ does not contain the possible plateau at ess sup $u$. Alternately, we can interpret $n \omega_{n}^{1 / n} F(t)^{1 / n^{\prime}}$ as the perimeter of the level set $\{u>t\}$ and apply the coarea formula to (5.2) to obtain

$$
\left\|u-u^{\star} \circ \tau\right\|_{q}^{q} \leq K_{n} \int F^{s}(u)^{\frac{1}{n}}\left|\nabla u^{q}\right| d x
$$

if $C$ has finite measure, this implies (1.4) with $p=1$.

Proof of Theorem 2. Let $\xi_{\infty}$ and $\tau$ be as in the proof of Theorem 1, and consider Lemma 3.1 with $\Psi(t)=t^{p}$. Since the intersection between any pair of balls decreases with the distance of their centers, it follows that

$$
\left\|u-u^{\star} \circ \tau\right\|_{p}^{p} \leq\left\|u^{\star}-u^{\star} \circ \tilde{\tau}\right\|_{p}^{p} \leq\left\|\nabla u^{\star}\right\|_{p} \cdot\|D \xi\|,
$$


where $\tilde{\tau}(x)=x-\|D \xi\| w$ for some unit vector $w$. The bound on the total variation of $\xi$ in (2.5) yields the claim.

Proof of Theorem 3. Let $\xi_{\infty}$ and $\tau$ be as in the proof of Theorem 1 . Since $u(x)=u^{\star}\left(x-\xi_{u(x)}\right)$ and $\left(u^{\star} \circ \tau\right)(x)=u^{\star}\left(x-\xi_{\infty}\right)$, Morrey's inequality says that

$$
\left|u(x)-\left(u^{\star} \circ \tau\right)(x)\right| \leq\left. M_{n, p}|| \nabla u\right|_{p} \cdot\left|\xi_{u(x)}-\xi_{\infty}\right|^{1-\frac{n}{p}} .
$$

The claim follows with (2.5).

\section{Dirichlet-Type functionals on $B V_{\text {loc }}$}

At last, we turn to more general convex gradient functionals. A Young function is a nonnegative, nondecreasing convex function $\Phi$ on $\mathbb{R}_{+}$with $\Phi(0)=0$. The Dirichlet functional associated with this Young function is defined by

$$
\mathscr{F}(u)=\int \Phi(|\nabla u|) d x
$$

provided that the distributional gradient of $u$ is locally integrable. If $\Phi$ grows linearly at infinity, the functional is extended to $B V_{\text {loc }}$ by

$$
\mathscr{F}(u)=\int \Phi(|\nabla u|) d x+\phi\left\|D^{s} u\right\|,
$$

where $\phi=\lim _{t \rightarrow \infty} \Phi(t) / t$, and $\left\|D^{s} u\right\|$ is the singular part of the total variation. Then $\mathscr{F}(u)$ is always well-defined but it may take the value $+\infty$. In this setting, the Pólya-Szegö inequality says that

$$
\mathscr{F}\left(u^{\star}\right) \leq \mathscr{F}(u)
$$

for all $u \in B V_{\text {loc }}[8]$.

Corollary 6.1. Let $\mathscr{F}$ be a Dirichlet functional on $\mathbb{R}^{n}$ given by a strictly increasing Young function $\Phi$. Let $u \in B V_{\text {loc }}$ be a nonnegative function that vanishes at infinity, and let $u^{\star}$ be its symmetric decreasing rearrangement. If

$$
\mathscr{F}(u)=\mathscr{F}\left(u^{\star}\right)<\infty,
$$

then there exists a translation $\tau$ such that

$$
\int \Psi\left(\left|u-u^{\star} \circ \tau\right|\right) d x \leq K_{n}\left(\int \Psi(u) d x\right)^{1 / n^{\prime}}\left(\int_{C_{\Phi}} \Psi(u) d x\right)^{1 / n}
$$

for every Young function $\Psi$ such that $\Psi \circ u$ is integrable. Here,

$$
C_{\Phi}=\left\{x \in \mathbb{R}^{n}: 0<u(x)<\operatorname{ess} \sup u,|\nabla u(x)| \in\{0\} \cup V\right\},
$$

and $V$ is the maximal open subset of $\mathbb{R}_{+}$such that $\Phi$ is affine on each connected component of $V$. The constant is given by $K_{n}=2 \omega_{n-1} / \omega_{n}$. 
Note that the conclusion depends on the Young function $\Phi$ only through the set $V$; in particular, if $\Phi$ is strictly convex then $V=\emptyset$ and $C_{\Phi}=C$.

Proof of Corollary 6.1. Cianchi and Fusco established in [9] that (2.1) holds under the given assumptions, i.e., the level sets of extremals are balls. Moreover, (2.2) holds for a.e. $t \in(0, \operatorname{ess} \sup u)$ such that $\left|\nabla u^{\star}\right|_{\rfloor \partial\left\{u^{\star}>t\right\}} \notin V$, i.e., $|\nabla u|$ is equidistributed on $\partial\{u>t\}$ for $\mathcal{H}^{n-1}$ almost every $x \in \partial\{u>t\}$. Let

$$
S_{\Phi}=S \cup\left\{t>0: F^{\prime}(t)=0\right\} \cup\left\{t>0:\left|\nabla u^{\star}\right|_{\lrcorner \partial\left\{u^{\star}>t\right\}} \in V\right\},
$$

and set $f(t)=\lambda_{1}\left((0, t) \backslash S_{\Phi}\right)$. Then $f \circ u^{\star}$ is absolutely continuous, its distribution function has no singular continuous component, and both $f \circ u^{\star}$ and $u^{\star}-f \circ u^{\star}$ are radially decreasing functions that vanish at infinity. By definition, $C_{\Phi}=u^{-1}\left(S_{\Phi}\right)$. Since

$$
\nabla(f \circ u)=\mathcal{X}_{C_{\Phi}} \nabla u,
$$

the set of critical points of $f \circ u$ is given by $C_{\Phi}$. Furthermore,

$$
\mathscr{F}(u)=\mathscr{F}(f \circ u)+\mathscr{F}(u-f \circ u),
$$

and correspondingly for $u^{\star}$. The Pólya-Szegö inequality holds for each summand, and therefore $f \circ u$ and $u-f \circ u$ must be extremals. In particular, $f \circ u$ satisfies (2.4). Since every level set $\{u>t\}$ with $t \notin S_{\Phi}$ is also a level set of $f \circ u$, it follows that

$$
\left|\xi_{t}-\xi_{\infty}\right| \leq\left(\frac{F_{\Phi}^{s}(t)}{\omega_{n}}\right)^{\frac{1}{n}}
$$

where

$$
F_{\Phi}^{s}(t)=\lambda_{n}\left(\left\{x \in C_{\Phi}: u(x)>t\right\}\right) .
$$

By Lemma 3.2 and Hölder's inequality,

$$
\begin{aligned}
\int \Psi\left(\left|u-u^{\star} \circ \tau\right|\right) d x \leq K_{n} \int_{0}^{\infty} F(t)^{\frac{1}{n^{\prime}}} F_{\Phi}^{s}(t)^{\frac{1}{n}} \Psi^{\prime}(t) d t \\
\quad \leq K_{n}\left(\int_{0}^{\infty} F(t) \Psi^{\prime}(t) d t\right)^{\frac{1}{n^{\prime}}}\left(\int_{0}^{\infty} F_{\Phi}^{s}(t) \Psi^{\prime}(t) d t\right)^{\frac{1}{n}} .
\end{aligned}
$$

Using the layer-cake principle, we recognize the integrals in the last line as $\int \Psi(u) d x$ and $\int_{C_{\Phi}} \Psi(u) d x$. As in Theorems 1-3, we can equivalently replace $u$ with $u^{\star}$ in these integrals and in the definition of $C_{\Phi}$.

If $u$ is supported on a set of finite measure, then we can use (6.3) with $\Psi(t)=t$ and apply Jensen's inequality once more to conclude 
that

$$
\begin{aligned}
\left\|u-u^{\star} \circ \tau\right\|_{1} & \leq\|\nabla u\|_{1} \cdot\left(\frac{\lambda_{n}\left(C_{\Phi}\right)}{\omega_{n}}\right)^{\frac{1}{n}} \\
& \leq \Phi^{-1}\left(\frac{\mathscr{F}(u)}{\lambda_{n}(\operatorname{supp} u)}\right) \cdot \lambda_{n}(\operatorname{supp} u)\left(\frac{\lambda_{n}\left(C_{\Phi}\right)}{\omega_{n}}\right)^{\frac{1}{n}} .
\end{aligned}
$$

Since $C_{\Phi} \subset \operatorname{supp} u$, this implies [9, Theorem 1.1]. For $\Phi(t)=t^{p}$, we recover (1.2) with $L_{n}=\omega_{n}^{-1 / n}$.

Corollary 6.2. Under the assumptions of Corollary 6.1, if $C_{\Phi}$ has finite measure, then there exists a translation $\tau$ such that

$$
\int \Phi\left(\left|u-u^{\star} \circ \tau\right| \cdot\left(\frac{\lambda_{n}\left(C_{\Phi}\right)}{\omega_{n}}\right)^{-\frac{1}{n}}\right) d x \leq \mathscr{F}(u) .
$$

Proof. Let $\xi_{\infty}$ and $\tau$ be as in the proof of Theorem 1. We will show that

$$
\int \Psi\left(\left|u-u^{\star} \circ \tau\right|\right) d x \leq \int \Psi(|\nabla u| \cdot|| D \xi||) d x
$$

for every Young function $\Psi$ such that the right hand side is finite. We then set $\Psi(t)=\Phi(t /\|D \xi\|)$, and use that

$$
\|D \xi\| \leq\left(\frac{\lambda_{n}\left(C_{\Phi}\right)}{\omega_{n}}\right)^{\frac{1}{n}}
$$

by (6.2).

For (6.3), we combine (3.3) with Lemma 3.1 and argue as in the proof of Theorem 2 that the integral on the left hand side increases if $u$ is replaced by $u^{\star} \circ \tilde{\tau}$, where $\tilde{\tau}(x)=x-\|D \xi\| w$ for some unit vector $w$. Since

$$
u^{\star}(x)-u^{\star} \circ \tilde{\tau}(x)=\int_{0}^{1}\left\langle\nabla u^{\star}(x+\theta\|D \xi\| w),\|D \xi\| w\right\rangle d \theta
$$

Jensen's inequality implies that

$$
\begin{aligned}
\int \Psi\left(\left|u^{\star}-u^{\star} \circ \tilde{\tau}\right|\right) d x & \leq \int \Psi\left(\int_{0}^{1}\left|\nabla u^{\star}(x+\theta|| D \xi|| w)\right| d \theta \cdot \| D \xi||\right) d x \\
& \leq \int \Psi\left(\left|\nabla u^{\star}\right| \cdot|| D \xi||\right) d x \\
& \leq \int \Psi(|\nabla u| \cdot|| D \xi||) d x
\end{aligned}
$$

The last step holds by the Pólya-Szegő inequality in (6.1). 


\section{REFERENCES}

[1] Frederick J. Almgren, Jr. and Elliott H. Lieb. Symmetric decreasing rearrangement is sometimes continuous. J. Amer. Math. Soc., 2(4):683-773, 1989.

[2] Angelo Alvino, Vincenzo Ferone, Guido Trombetti, and Pierre-Louis Lions. Convex symmetrization and applications. Ann. Inst. H. Poincaré Anal. Non Linéaire, 14(2):275-293, 1997.

[3] Luigi Ambrosio, Nicola Fusco, and Diego Pallara. Functions of Bounded Variation and Free Discontinuity Problems. Oxford Mathematical Monographs. The Clarendon Press Oxford University Press, New York, 2000.

[4] F. Brock. Weighted Dirichlet-type inequalities for Steiner symmetrization. Calc. Var. Partial Differential Equations, 8(1):15-25, 1999.

[5] John E. Brothers and William P. Ziemer. Minimal rearrangements of Sobolev functions. J. Reine Angew. Math., 384:153-179, 1988.

[6] A. Burchard. Steiner symmetrization is continuous in $W^{1, p}$. Geom. Funct. Anal., 7(5):823-860, 1997.

[7] Andrea Cianchi and Adele Ferone. On symmetric functionals of the gradient having symmetric equidistributed minimizers. SIAM J. Math. Anal., 38(1):279-308, 2006.

[8] Andrea Cianchi and Nicola Fusco. Functions of bounded variation and rearrangements. Arch. Ration. Mech. Anal., 165(1):1-40, 2002.

[9] Andrea Cianchi and Nicola Fusco. Minimal rearrangements, strict convexity and critical points. Appl. Anal., 85(1-3):67-85, 2006.

[10] Lawrence C. Evans and Ronald F. Gariepy. Measure Theory and Fine Properties of Functions. Studies in Advanced Mathematics. CRC Press, Boca Raton, FL, 1992.

[11] Adele Ferone and Roberta Volpicelli. Minimal rearrangements of Sobolev functions: a new proof. Ann. Inst. H. Poincaré Anal. Non Linéaire, 20(2):333-339, 2003.

[12] Adele Ferone and Roberta Volpicelli. Convex rearrangement: equality cases in the Pólya-Szegő inequality. Calc. Var. Partial Differential Equations, 21(3):259-272, 2004.

[13] Bernhard Kawohl. Rearrangements and Convexity of Level sets in PDE. Lecture Notes in Mathematics, Vol. 1150. Springer-Verlag, Berlin, 1985.

[14] Elliott H. Lieb and Michael Loss. Analysis. Graduate Studies in Mathematics, Vol. 14. American Mathematical Society, Providence, RI, 1997.

[15] George Pólya and Gabor Szegő. Isoperimetric Inequalities in Mathematical Physics. Annals of Mathematics Studies, no. 27. Princeton University Press, Princeton, N. J., 1951.

[16] Giorgio Talenti. A weighted version of a rearrangement inequality. Ann. Univ. Ferrara Sez. VII (N.S.), 43:121-133 (1998), 1997.

Department of Mathematics - University of Toronto, 40 St. George

Street, Toronto, Canada M5S 2E4

E-mail address: almut@math.toronto.edu

Dipartimento di Matematica - Seconda Università di Napoli, Viale

Lincoln 5, 81100 CASERTA, ItAly

E-mail address: adele.ferone@unina2.it 